Ciencia y Educación, Vol. 5, No. 3, septiembre-diciembre, 2021

ISSN (impreso): 2613-8794・ISSN (en línea): 2613-8808

DOI: https://doi.org/10.22206/cyed.2021.v5i3.pp79-95

\title{
Racismo implícito en grupo de estudiantes del Instituto Tecnológico de Santo Domingo
}

\author{
Implicit racism in an Instituto Tecnológico de \\ Santo Domingo student group
}

Larissa De Peña ${ }^{a}$ ORCID: 0000-0002-4685-6782

Viviana De León ${ }^{\mathrm{b}}$ ORCID: 0000-0001-9901-5912

Recibido: 25/01/2021・Aprobado: 28/06/2021

Cómo citar: De Peña Hernández, L. M. (2021). Racismo implícito en grupo de estudiantes del Instituto Tecnológico de Santo Domingo. Ciencia y Educación, 5(3), 79-95. Doi: https://doi.org/10.22206/cyed.2021.v5i3.pp79-95

\section{Resumen}

Esta investigación tiene como objetivo evaluar si existen manifestaciones de racismo implícito en un grupo de estudiantes del Instituto Tecnológico de Santo Domingo _-INTEC_- analizando si hay diferencias según su sexo, religiosidad, identidad política y clase social. Es de gran importancia porque concientiza sobre este fenómeno difícil de identificar, pero muy perjudicial para el estudiantado y la sociedad. Es de enfoque cuantitativo, alcance descriptivo y diseño no experimental transeccional. Se contó con una muestra de 70 estudiantes del INTEC — seleccionada por muestreo no probabilístico- de distintos sexos, clases sociales, niveles de religiosidad e identidades políticas. El instrumento utilizado fue el Test de asociación implícita, elaborado por Greenwald y Banaji en 1998. Los resultados indican que sí se presentó racismo implícito, principalmente en distintos grados de preferencia automática hacia personas blancas sobre negras, sin diferencias significativas según el sexo, la religiosidad, la identidad política, ni la clase social de este grupo de estudiantes.

Palabras clave: discriminación étnica, prejuicio racial, grupo étnico, cultura, xenofobia.

\begin{abstract}
This research aims to assess if there are manifestations of implicit racism in a group of students of the Instituto Tecnológico de Santo Domingo _-INTEC_-, analyzing if there are differences according to their gender, religiosity, political identity and social class. It is very important because it raises awareness about this phenomenon, which is difficult to identify, but very harmful for students and society. It has a quantitative approach, descriptive scope and non-experimental transectional design. A sample of 70 INTEC students — selected by non-probabilistic sampling - of different sexes, social classes, levels of religiosity and political identities was used. The instrument used was the Implicit Association Test, developed by Greenwald and Banaji in 1998. The results indicate that implicit racism was present, mainly in different degrees of automatic preference for whites over blacks, with no significant differences according to sex, religiosity, political identity or social class of this group of students.
\end{abstract}

Keywords: Ethnic discrimination, racial prejudice, ethnic group, culture, xenophobia.

\footnotetext{
a Investigadora independiente, República Dominicana. Correo-e: larissadepena@gmail.com

b Investigadora independiente, República Dominicana. Correo-e: 1087480@est.intec.edu.do
} 


\section{Introducción}

A lo largo de la historia ha existido el racismo, por el que las personas son discriminadas por sus rasgos físicos o culturales. Dicló (2019) señala que, en República Dominicana, a pesar de este ser un país con una mezcla simbiótica de diversos grupos étnicos, desde los tiempos de la colonización se han cometido actos inconcebibles basados en el racismo, el cual se ha mantenido a través de los años.

En la actualidad se podría pensar que las personas racistas son la minoría y que esta práctica se ha reducido considerablemente; esto es correcto si el enfoque está en el racismo explícito, que anteriormente ocurría de maneras más extremas. Sin embargo, Pascale (2010) indica que aún se mantiene la discriminación en gran magnitud, pero ahora principalmente de una manera más sutil y difícil de identificar.

Según Pascale (2010), en Latinoamérica los y las afrodescendientes cuentan con los peores indicadores de ingreso y educación de la población. La Comisión Económica para América Latina y el Caribe - CEPAL - (2016) indica que en países de Latinoamérica —como Ecuador, Brasil, Perú y Uruguaydentro de la población ocupada, la escolaridad media de no afrodescendientes — 9.4 años — es mayor en 2.3 años que la de afrodescendientes — 7.1 años_- y en la tasa de ingresos la proporción de población afrodescendiente que se ubica en el grupo de más bajos ingresos es del 34\%; mientras que de no afrodescendientes es de un 19\%. Marques-García (2019) señala que en República Dominicana, según un informe de la Oficina de Desarrollo Humano - $\mathrm{ODH}$ - para el Ministerio de Economía, Planificación y Desarrollo - MEPyD_las personas de descendencia haitiana cuentan con una situación social muy inferior a la del $20 \%$ con menos recursos de dominicanos y dominicanas.

El racismo de estos tiempos es un problema muy complejo. Mato (2017) expresa que su presencia en Latinoamérica hace que las inequidades de estos países sean ética y jurídicamente cuestionables, provocando que no haya desarrollo ni mejora en la calidad de vida de las sociedades.

Esta investigación es de gran importancia, ya que concientiza sobre la nueva manera en que se manifiesta predominantemente el racismo en la actualidad. Vemos que hoy en día en América Latina se expresan las actitudes racistas de una manera más difusa (París, 2002). A su vez, es necesario que esto se reconozca porque este racismo es más peligroso para las sociedades. Vinueza (1999) indica que posiblemente sea más nocivo por estar oculto y presentarse como algo natural.

Es fundamental que a través de este estudio las personas entiendan que este racismo es inculcado y que con la educación se pueden hacer muchos cambios para que desaparezca, lo cual es vital por la gran cantidad de consecuencias negativas que tiene para las víctimas. Provoca que estas asuman para sí mismas los insultos que les arrojan las personas que las discriminan — por exclusión, humillaciones, ofensas o bromas relacionadas a su etnia-, creando en ellas sentimientos de inferioridad (Pineda, 2018b).

\section{Racismo}

El racismo es una ideología basada en la desigualdad de los grupos étnicos en los seres humanos. Es visible a través de actos de discriminación que vienen a raíz de prejuicios y estereotipos (Aguayo \& Piña, 2015). Chirix \& Sajbin (2019) sugieren que es una manifestación de perturbación y se fundamenta en una idea de superioridad frente al otro o la otra.

La ideología racista conlleva un trato diferente hacia ciertos sectores sociales definidos por rasgos culturales, biológicos o fenotípicos, reales o imaginarios. Por medio de las prácticas discriminatorias, el racismo parece expandirse en todas las instituciones sociales modernas: la vivienda, la escuela, la empresa, el sindicato, la policía, entre otros (París, 2002).

Además de poder ser entendido desde su dimensión ideológica, el racismo también tiene sus funciones 
prácticas, en las que se puede visualizar desde la acción social. En las sociedades en que esto ocurre, la transparencia del racismo, desde el punto de vista cognitivo, genera un conjunto de reglas para conocer y socializar con el otro o la otra (Hellebrandová, 2014).

\section{Racismo implícito}

Sin importar los grandes esfuerzos por la eliminación del racismo, la crisis económica de hoy en día y la época digital, que ha facilitado la transmisión de ideas de manera masiva, han llevado a que este se mantenga desde expresiones más sutiles (Buraschi \& Aguilar-Idáñez, 2017). Según Pascale (2010), los cambios de normativas sociales en los últimos 50 años, en que se castigan manifestaciones de racismo, han culminado en la aparición de formas más encubiertas que evidencian los prejuicios existentes hacia las víctimas, dando lugar a un racismo implícito. Espelt \& Javaloy (1997) señalan que las desigualdades que se visualizan en la distribución del poder socioeconómico y político demuestran que existe un racismo que puede ser más dócil en cuanto a sus manifestaciones, pero no en sus consecuencias; estas incluyen marginación en el trabajo, la vivienda, la escuela y la sociedad para las minorías étnicas.

El nuevo racismo involucra el desplazamiento de las diferencias fenotípicas hacia las culturales, donde se cambia el deseo hacia la pureza racial por el anhelo a la identidad cultural auténtica. Además, se observa un desplazamiento de la desigualdad hacia la diferencia, en que el desprecio por inferiores se modifica por la evitación del otro o de la otra (Espelt \& Javaloy, 1997). Pineda (2018a) señala que una de las formas del racismo de hoy es el racismo simbólico o moderno, que se expresa por medio de conductas simbólicas que incluyen sensaciones de que afroamericanos y afroamericanas están violando valores importantes o haciendo demandas ilegítimas de cambios en el estatus social.

Una de las actitudes que adopta el racismo moderno es el rechazo de los estereotipos y de la discriminación descarada. Un ejemplo de esta es que en la actualidad pocos o pocas dicen que las personas negras son más perezosas; sin embargo, indican que las blancas son más ambiciosas. Otra actitud consiste en la oposición al cambio racial por razones no raciales; las personas blancas, en su cotidianidad, rechazan ideas que colaboren para la eliminación del racismo (Espelt \& Javaloy, 1997).

Espelt \& Javaloy (1997) indican que el sentimiento de amenaza basado en el grupo constituye otra de las actitudes racistas que son motivadas por sentir que se ignora el grupo propio. Observamos que hay cierto recelo con respecto a ayudas especiales que se realizan a las minorías, como cuotas en los empleos o en la educación. Adicionan las concepciones individualistas del éxito en el trabajo; se piensa que si las personas negras no mejoran la situación en que viven es debido a que no trabajan lo suficiente. Se convierte a la víctima en culpable y se niega la existencia del racismo.

Estos autores explican que de estas actitudes se deriva la conformidad con las nuevas formas sin adoptarlas de manera interna en su totalidad. También, se suman pequeñas agresiones indirectas y conductas de evitación (Espelt \& Javaloy, 1997).

Además, ha surgido el infraracismo, un discurso muy general que se reconoce por no ser intencional — se oculta el racismo en el comentario-. También está el racismo automático, que ha sido naturalizado por la cultura y no es conocido o definido como atribución de valor o conjunto de representaciones ideológicas (Pineda, 2018a). Pascale (2010) explica otros conceptos del nuevo racismo: "Mientras la hetero-etnización representa la exagerada atribución de diferencias culturales al exogrupo, la ontologización atribuye más rasgos naturales que culturales al exogrupo y la infra-humanización niega la capacidad del exogrupo de expresar emociones secundarias" (pp. 65-66).

El racismo axiológico es aquel que se manifiesta a través de un conjunto de valores y creencias que asigna predicados negativos o positivos a las personas en función de su color de piel. El racismo acostumbrado es el conjunto de actitudes, reacciones o comentarios que provienen de los prejuicios o de la falta de información (Pineda, 2018a). 
Pascale (2010) indica que el racismo ambivalente es detonado a partir de un conflicto emocional que trae consigo tanto sentimientos positivos como negativos hacia ciertos grupos raciales. También encontramos el racismo aversivo, caracterizado por un pensamiento de igualdad de grupos étnicos; no obstante, viene con actitudes de evitación hacia cierto grupo étnico, principalmente cuando se tiende a relacionar la conducta de las víctimas con elementos relacionados a su etnia.

\section{Origen histórico del racismo en República Dominicana}

El racismo en América Latina constituye una consecuencia de la colonización que llevaron a cabo potencias europeas. Esta minoría dominante, con ambiciones expansionistas, justificó el maltrato y sometimiento de indígenas, africanos y africanas. La guerra escenificada por los imperios europeos y la iglesia cristiana tenía como objetivo hacer legal la subordinación y la transformación de los indios y los negros en esclavos, por medio de discursos en la misión religiosa y la superioridad natural de los europeos (Pineda, 2018b).

Según Montero (2019), se manifestaban relaciones de superioridad de grupos étnicos, que venían como un hecho inseparable al proceso de represión y exterminio de la población aborigen, lo cual se ve claramente en el trato brutal a que fue sometida la población autóctona. Seda-Prado (2003) especifica que enfermedades, el trabajo minero y de plantación, y otras causantes provocaron que se redujera la población indígena y se trajera mano de obra africana a la isla.

Seda-Prado (2003) señala que en períodos siguientes continuó el rechazo tanto de afrodescendientes como de nuestras raíces étnicas en República Dominicana. Intelectuales del período colonial en el siglo XX escondían nuestras raíces negras-africanas, lo que tomó mayor fuerza durante la dictadura de Trujillo y se observa en las escrituras de Manuel Arturo Peña Batlle y Joaquín Balaguer.

Desde la era colonial, la clase gobernante de la República Dominicana ha generado un sentimiento antinegro, expresamente antihaitiano, donde se observa segregación y explotación política del miedo. El antihaitianismo se formó en una ideología estatal durante la dictadura Trujillo, quien en 1937 llevó a cabo un exterminio de la población haitiana, donde fallecieron más de 30,000 personas (Restrepo, 2019).

Además, Trujillo generó una estrategia de lo que pretendió ser una limpieza étnica que consistía en obligar a la pronunciación de la palabra perejil, una palabra difícil de pronunciar para personas que vienen de Haití. Hoy en día, vemos una técnica parecida en que la policía utiliza el color de la piel y el acento como punto de referencia para solicitar o rechazar documentos de identidad de quienes consideran haitianos o haitianas (Restrepo, 2019). Denis-Rosario \& Paulino (2016) explican que aquellas personas que son dominicanas pero de ascendencia haitiana en nuestro país viven la situación de no tener nacionalidad, por lo que parecen ser nada ante la ley.

\section{Factores relacionados al racismo}

Racismo y cultura. Cisneros (2001) señala que el racismo se refiere a una ideología y un comportamiento social que se caracteriza por la jerarquización de personas basándose en sus rasgos físicos y naturales, pero además en sus manifestaciones culturales. Cea (2009) explica que existe un racismo denominado racismo cultural que tiene lugar principalmente en ambientes donde hay inmigración, ya que trae consigo no solo nuevas diferencias fenotípicas, sino también socioculturales, por lo cual en estos casos se ve la necesidad de utilizar otros conceptos, además del racismo.

La discriminación, la segregación, la marginación y el racismo son formas extremas de intolerancia cultural. A través del término de racismo se infiere que las acciones históricas y manifestaciones culturales se determinan por medio de los distintos grupos étnicos y que existe una superior a las otras (Cisneros, 2001).

Racismo y sexo. Wade (2013) destaca que el grupo étnico y el género nacen por medio de la relación con otra persona, explicando que estos no son 
aspectos de experiencia distintos, están interconectados. Añade que las jerarquías raciales surgen con las relaciones de sexo/género y entender esto permite explorar más a profundidad en lugar de permanecer como un simple discurso.

La mujer afroamericana es discriminada por dos razones: por ser mujer y por ser negra. Durante los años de esclavitud fue marginada tanto por hombres blancos como por negros; siendo los hombres afroamericanos quienes lucharan por su libertad, dejando de lado a la mujer — por ser considerada inferior- y siendo los blancos quienes las excluían, tanto por su etnia como por su sexo (Romero, 2015).

La práctica de definir quién pertenecía genealógicamente a las familias provocó que se prestara mucha atención a la pureza de sangre. El proceso de colonización en América trasladó esto a constituir un elemento racial, haciendo que ser negra significara impureza o falta de limpieza de sangre. El honor iba de mano con la propiedad sexual de las mujeres, ya que influía en la pureza de sangre que estas podían garantizar (Wade, 2013).

Quintero (2013) señala que llevó a cabo un estudio sobre racismo y discriminación en estudiantes universitarios de Bogotá y París. En este se encontró una notoria mayor cantidad de mujeres que habían sido víctimas de situaciones de racismo que de hombres, donde de las 775 situaciones relatadas, el $71 \%$ fueron vividas por mujeres.

Racismo y educación. El racismo y la discriminación en las escuelas se presentan principalmente en asuntos de gestión y administración, en el currículo y en prácticas de aula. En este ámbito, se visualizan desigualdades al momento de acceder a ciertos elementos o recursos y en el desempeño social, además en el aprendizaje, los resultados y los logros (Velasco-Cruz, 2016).

Soler (2018) señala que en las décadas de 1960 y 1970 surgen los primeros intentos de aplicación de enfoques educativos multiculturales, que tenían como objetivo promover la diversidad de las poblaciones, principalmente en Estados Unidos, Inglaterra, Canadá y Australia. Sin embargo, Baronnet \& Morales-González (2018) explican que estas prácticas dirigen la educación de manera compensatoria hacia la diferencia cultural y, a pesar de que promueven la tolerancia y el respeto, tienden a motivar a la ejecución de acciones que los mismos y las mismas estudiantes consideran discriminatorias; de este modo, se visualizan los derechos de manera individual y se institucionaliza la diferencia, desconociendo aquellos actos de oposición y resistencia.

Woldenberg (1997) explica que la educación permite hacer grandes avances para la erradicación de los prejuicios racistas. La construcción de igualdad formal podría y debería reflejarse en el proceso educativo. La noción de pluriculturalidad permite reconocer y valorar las distintas culturas, con el respeto que cada una merece. Esta idea ayudaría a combatir los privilegios y exclusivismos y se valoraría la diversidad.

Racismo en universidades. Aguayo \& Piña (2015) indican que investigaron tres espacios universitarios diferentes y observaron estudiantes que asociaban términos positivos hacia indígenas; no obstante, una quinta parte de estos y estas denotaba repulsión y prejuicio. Expresan que, a pesar de no ser la mayoría, dos de cada diez estudiantes rechazan a la persona de origen indígena; mostrando que se presenta racismo en las universidades.

Ortiz-Hernández et al. (2011) señalan que en su estudio sobre el racismo en la Universidad Autónoma Metropolitana de México se encontraron resultados que sostenían la existencia de prácticas discriminatorias ante estudiantes de piel oscura. Indican que estos tenían mayor probabilidad de ser marginados o marginadas y que esto se puede atribuir al eurocentrismo que predomina en México.

Analizar el racismo en el ambiente universitario es de gran relevancia a nivel social, ya que brinda información que facilita la comprensión de este como un hecho actual, incitando a que se busquen vías que colaboren con la erradicación de la problemática. La desegregación en escuelas y universidades en los Estados Unidos generó una respuesta pública de 
toma de iniciativas contra la discriminación; a pesar de esto, se vieron perjudicadas por la permanencia del racismo en universidades y centros de enseñanza (Quintero, 2014).

Racismo y clases sociales. El racismo y la xenofobia son, en gran cantidad de ocasiones, el reflejo de un fuerte clasismo en las personas. La discriminación hacia inmigrantes, que viene siendo una exclusión social, se fundamenta principalmente en la clase social, más que en la etnia o la cultura (Cea, 2009).

Cea (2009) señala que el racismo ordinario y el de clase convergen en diversos puntos. Una persona que es inmigrante puede ser excluida tanto por ser extranjera como por tener como origen un país pobre y formar parte de una clase social desfavorecida. Concluye que el nivel en que es aceptado un extranjero o una extranjera depende de sus condiciones económicas y académicas.

Ipar \& Giller (2016) señalan que en los inicios del racismo moderno no se apreciaba un racismo exclusivo con el que se buscara la eliminación de poblaciones completas de una etnia, sino un racismo inclusivo con el cual se creaban relaciones de poder y había explotación de clase. Por lo tanto, el grupo étnico y la clase representan dos elementos básicos relacionados con la división social del trabajo en la actualidad. Esta historia recae en un racismo de clase que busca crear diferencias en poblaciones para el establecimiento de los roles que se ejerzan en la división del trabajo.

Racismo e ideologías políticas liberales y conservadoras. El liberalismo político trata de eludir temas o tomar posición sobre asuntos filosóficos; busca evitar las polémicas y lograr que todos los ciudadanos y las ciudadanas estén de acuerdo con las orientaciones morales, religiosas o filosóficas más heterogéneas que puedan respaldar (Garreta, 2012).

Mazariegos (2006) expresa que una explicación aceptada por muchos y muchas es que lo que plantea el liberalismo resulta contradictorio cuando se trata de la ciudadanía de determinadas etnias, como la de los y las indígenas. Agrega que la ciudadanía es solo aceptada para personas civilizadas; por lo cual, un o una indígena no puede adquirir ciudadanía a menos que deje de ser el ser humano inculto primitivo imaginado por quienes se identifican como liberales.

Rottenbacher et al. (2011) explican que el conservadurismo es una ideología política por medio de la cual se manifiestan pensamientos de oposición frente a cambios sociales, siempre optando por opciones más tradicionales y confiables tanto para las instituciones y su manera de organizarse, como para el comportamiento de las personas, que viene con una propuesta de desigualdad entre ellas.

Las personalidades conservadoras tienen ciertas actitudes que denotan preferencia por aquellas acciones y decisiones jerárquicas donde se presentan diferencias entre el poder de determinados grupos e individuos; con esto viene también la preferencia a la superioridad de un miembro del grupo propio frente a otras personas externas a este. Además, existe en ellas una motivación por un pensamiento claro y preciso sobre el mundo social, que culmina en el rechazo a lo ambiguo, lo desconocido y lo que perturba el orden en la sociedad (Rottenbacher et al., 2011).

Racismo y religiosidad. Hall et al. (2010) indican que la identidad religiosa crea percepciones de la misma manera que la política, la nacional y otras identidades. Las personas religiosas son más propensas a responder a otros u otras basándose en si son parte de su grupo o no lo son; principalmente la doctrina religiosa que divide entre creyentes y no creyentes. Concluyen que quienes se identifican fuertemente con una religión son más propensos o propensas a humillar a personas que no pertenecen a su grupo.

Johnson et al. (2010) explican que existe una estrecha relación, muy compleja, entre diversas medidas de religiosidad y el prejuicio racial; una gran cantidad de dimensiones de la religión se correlacionan con este prejuicio - como la religión extrínseca y el fundamentalismo-, aunque otras no — como es el caso de la religión intrínseca y la búsqueda religiosa-. Hall et al. (2010) señalan que, en la medida en que la religión tienda a practicarse entre un determinado grupo étnico, personas de otras etnias pueden parecer 
que no pertenecen a estos grupos religiosos. Agregan que, por lo tanto, la idea de que una etnia representa alguna afiliación religiosa sirve para explicar el vínculo de la religiosidad y el racismo.

\section{Metodología}

\section{Objetivos}

\section{Objetivo general}

- Evaluar si existen manifestaciones de racismo implícito en un grupo de estudiantes del INTEC.

\section{Objetivos específicos}

- Identificar si se presentan diferencias con relación al sexo en la existencia o no de racismo implícito en un grupo de estudiantes del INTEC.

- Determinar si se observan diferencias en la presencia o no de racismo implícito en un grupo de estudiantes del INTEC por su religiosidad.

- Distinguir si se presentan diferencias en la existencia o no de racismo implícito en un grupo de estudiantes del INTEC según su identidad política.

- Analizar si hay diferencias en la existencia o no de racismo implícito en un grupo de estudiantes del INTEC según su clase social.

\section{Diseño}

La presente investigación tiene un enfoque cuantitativo, debido a que se basa en la búsqueda ordenada y precisa de datos fundamentados en la medición numérica y el análisis estadístico. Su alcance es descriptivo, ya que se pretende detallar las características principales del racismo implícito en un grupo de estudiantes del INTEC (Hernández et al., 2014).

Su diseño es no experimental transeccional descriptivo porque no se manipuló ninguna variable para investigar este fenómeno, pues los datos se recopilaron en un solo momento y, como se mencionó, se indagó sobre el racismo implícito en un grupo de estudiantes del INTEC para recopilar e informar sobre las características principales de este (Hernández et al., 2014).

\section{Muestra}

La muestra está constituida por 70 estudiantes del INTEC. Se llevó a cabo un muestreo no probabilístico, ya que las decisiones para su selección fueron basadas en características y propósitos específicos de la investigación, como la inclusión de distintas clases sociales, sexos, identidades políticas y niveles de religiosidad. Esto permitió analizar si había diferencias en la existencia o no de racismo implícito en este grupo de estudiantes del INTEC según estas variables (Hernández et al., 2014).

\section{Instrumento}

El instrumento utilizado fue el Test de asociación implícita, TAI, o IAT —en inglés—, ya que es una de las pruebas indirectas que permiten estudiar comportamientos implícitos más reconocidas. Fue creado por Anthony G. Greenwald y Mahzarin R. Banaji en 1998 (Ayala, 2012). Tiene una duración aproximada de 20 minutos y su confiabilidad oscila entre 0.70 y 0.90 ; en países de habla hispana, entre 0.75 y 0.93 (Ayala, 2012; Tosi et al., 2018).

Contiene siete ítems de asociación en que se presentan estímulos visuales y verbales para que los y las participantes los agrupen en categorías. El proceso se sintetiza en cinco pasos: aprendizaje de las categorías del objeto - personas blancas y negras-, aprendizaje de las categorías de los atributos - personas buenas y malas-, bloque compatible - se designa una tecla de respuesta para los estímulos de las categorías personas blancas y personas buenas, otra para personas negras y personas malas-; aprendizaje de cambio de localización espacial con respecto a las categorías del objeto - se invierte la ubicación de la categoría de los estímulos personas blancas y personas negras-; por último, bloque incompatible - se designa una tecla de respuesta para los 
estímulos de las categorías personas blancas y personas malas, otra para personas negras y personas buenas(Tosi et al., 2018).

Tosi et al. (2018) explican que el resultado, denominado efecto IAT, se obtiene mediante un proceso de puntuación denominado algoritmo $D$. De manera sintetizada, se calcula la diferencia en los promedios de respuesta de los bloques compatible e incompatible y se divide por la desviación estándar de las latencias para estas dos categorías.

Los resultados se presentan a través de la puntuación D. Aquellos positivos muestran una preferencia implícita por personas blancas frente a personas negras -Una puntación $\mathrm{D}$ mayor a 0.15 y menor o igual a 0.35 indica una leve preferencia; mayor a 0.35 y menor a 0.65 , moderada; y mayor o igual a 0.65 , fuerte- . Los resultados negativos indican una preferencia implícita por personas negras frente a blancas -Menor o igual a -0.65 , una fuerte preferencia; mayor a -0.65 y menor a -0.35 , moderada; y mayor o igual a -0.35 y menor a -0.15 , leve-. Una puntuación $D$ que se encuentre entre -0.15 y 0.15 indica ligera o ninguna preferencia racial automática (Greenwald et al., 1998).

\section{Procedimiento}

En primer lugar, se solicitó en el área de Ciencias Sociales y Humanidades del INTEC el uso de la cámara de Gesell de la universidad —habitación dividida en dos espacios por un vidrio de visión unilateral, que disminuye la influencia del observador sobre lo que se está analizando-; esta se utilizó porque el instrumento requiere de la eliminación de cualquier distractor externo debido a que involucra la velo- cidad de la respuesta y la cantidad de errores para el análisis de resultados.

Luego de haber recibido la autorización, se buscaron en el campus de la universidad posibles participantes de distintos sexos y distintas identidades políticas, clases sociales y niveles de religiosidad con el fin de posteriormente analizar si estas variables tenían relación con los resultados obtenidos. Se les dio a conocer información sobre la investigación, el tipo de prueba a la que se someterían y el día en que se llevaría a cabo.

Después de conseguir 70 personas dispuestas a ser parte del estudio, se les informó sobre el acuerdo de confidencialidad de la prueba y su duración. También se les explicó cómo debían interpretar los resultados, debido a que el test puede arrojar información delicada, como indicar que una persona tiene prejuicios raciales, a pesar de que esta no lo considere así.

Se tomaron dos días para aplicar el Test de asociación implícita, en los cuales se reunieron en la cámara de Gesell del INTEC a los voluntarios y las voluntarias a participar en la investigación. Por turnos, se les impartió la prueba mediante el uso de dos computadoras en las que se registraron los resultados de cada participante, junto a sus datos sociodemográficos, para luego analizarlos.

\section{Resultados}

A continuación, en la Tabla 1 se observa la preferencia racial automática que presenta la muestra de este estudio, con sus respectivas frecuencias y porcentajes. 
Tabla 1

Tabla de frecuencias: preferencia racial automática

\begin{tabular}{|c|c|c|c|c|}
\hline Preferencia Racial Automática & Frecuencia & Porcentaje & Porcentaje válido & $\begin{array}{l}\text { Porcentaje } \\
\text { acumulado }\end{array}$ \\
\hline $\begin{array}{c}\text { Fuerte preferencia automática hacia } \\
\text { personas negras sobre personas } \\
\text { blancas }\end{array}$ & 1 & 1.429 & 1.429 & 1.429 \\
\hline $\begin{array}{c}\text { Preferencia automática moderada } \\
\text { hacia personas negras sobre personas } \\
\text { blancas }\end{array}$ & 3 & 4.286 & 4.286 & 5.714 \\
\hline $\begin{array}{l}\text { Leve preferencia automática hacia } \\
\text { personas negras sobre personas } \\
\text { blancas }\end{array}$ & 5 & 7.143 & 7.143 & 12.857 \\
\hline $\begin{array}{l}\text { Ligera o sin preferencia automática } \\
\text { hacia personas blancas sobre personas } \\
\text { negras }\end{array}$ & 15 & 21.429 & 21.429 & 34.286 \\
\hline $\begin{array}{c}\text { Leve preferencia automática hacia } \\
\text { personas blancas sobre personas } \\
\text { negras }\end{array}$ & 14 & 20.000 & 20.000 & 54.286 \\
\hline $\begin{array}{l}\text { Preferencia automática moderada } \\
\text { hacia personas blancas sobre personas } \\
\text { negras }\end{array}$ & 19 & 27.143 & 27.143 & 81.429 \\
\hline $\begin{array}{c}\text { Fuerte preferencia automática hacia } \\
\text { personas blancas sobre personas } \\
\text { negras }\end{array}$ & 13 & 18.571 & 18.571 & 100.000 \\
\hline Missing & 0 & 0.000 & & \\
\hline Total & 70 & 100.000 & & \\
\hline
\end{tabular}

Nota: la fuente es el Programa JASP — a partir de datos obtenidos de TAI aplicado a un grupo de estudiantes del INTEC en 2020—.

En la Tabla 1 se puede observar que la mayoría de los y las participantes de la muestra tiene una preferencia automática moderada hacia personas blancas sobre personas negras, representando un $27.143 \%$ de la muestra; le siguen aquellos y aquellas con ligera o ninguna preferencia automática hacia personas blancas sobre personas negras, un $21.429 \%$; después, con leve preferencia automática hacia personas blancas sobre personas negras, un 20.000\%; luego, con fuerte preferencia automática hacia personas blancas sobre personas negras, un $18.571 \%$; le siguen aquellos y aquellas con leve preferencia automática hacia personas negras sobre personas blancas, un 7.143\%.; luego, con preferencia automática moderada hacia personas negras sobre personas blancas, un $4.286 \%$; por último, quienes presentan fuerte preferencia automática hacia personas negras sobre personas blancas, un $1.429 \%$.

En la Tabla 2 se visualizan los descriptivos de la puntuación $D$-que indica preferencia racial automática- que presentan los y las estudiantes de sexo femenino y masculino de la muestra, indicándose tamaño de muestra, media, desviación y error estándar de cada grupo. 
Tabla 2

Descriptivos de puntuación D

\begin{tabular}{cccccc}
\hline & Grupo & N & Media & SD & SE \\
\hline $\begin{array}{c}\text { Puntuación } \\
\text { D }\end{array}$ & Femenino & 26 & 0.262 & 0.399 & 0.078 \\
\hline & & & & & \\
\hline
\end{tabular}

Nota: la fuente es el Programa JASP — a partir de datos obtenidos de TAI aplicado a un grupo de estudiantes del INTEC en 2020 -

En la Tabla 2 se observa que la media de la puntuación $D$ en los hombres es de 0.329 y en las mujeres de 0.262 .

A continuación, en la Tabla 3 se observa la prueba $\mathrm{T}$ de las diferencias de medias para la variable de puntuación $D$ de los y las estudiantes del sexo femenino y masculino de la muestra, que indica si existen diferencias significativas entre las medias de la puntuación $D$ de ambos grupos.

\section{Tabla 3}

Prueba $T$ de diferencias de medias para la variable de puntuación $D$

\begin{tabular}{cccc}
\hline & $\mathbf{t}$ & $\mathbf{d f}$ & $\mathbf{p}$ \\
\hline Puntuación D & -0.664 & 68.000 & 0.509 \\
\hline
\end{tabular}

Nota: la fuente es el Programa JASP — a partir de datos obtenidos de TAI aplicado a un grupo de estudiantes del INTEC en 2020 -

En la Tabla 3 se puede apreciar que el valor de $\mathrm{p}$ es igual a 0.509 . Al ser el valor de P mayor a 0.05, se interpreta que no existen diferencias significativas entre la puntuación $D$ de hombres y mujeres.

En la Tabla 4 se visualizan los descriptivos de la puntuación $D$ - que indica preferencia racial automática- que presentan los y las estudiantes de la muestra de distintas religiosidades, mostrándose media, desviación estándar y tamaño de cada grupo.

\section{Tabla 4}

Descriptivos de puntuación D

\begin{tabular}{cccc}
\hline Religiosidad & Media & SD & N \\
\hline No religioso/a & 0.191 & 0.472 & 29 \\
Ligeramente religioso/a & 0.418 & 0.353 & 22 \\
Moderadamente religioso/a & 0.355 & 0.345 & 18 \\
Fuertemente religioso/a & 0.168 & & 1 \\
\hline
\end{tabular}

Nota: la fuente es el Programa JASP — a partir de datos obtenidos de TAI aplicado a un grupo de estudiantes del INTEC en 2020 -

En la Tabla 4 se observa que la media de la puntuación $D$ en quienes se identifican como ligeramente religiosos o religiosas es de 0.418 ; los moderadamente religiosos o religiosas tienen una media de 0.355 ; los no religiosos o religiosas tienen una media de $0.191 \mathrm{y}$ aquellos/as fuertemente religiosos o religiosas poseen una media de 0.168 .

A continuación, en la Tabla 5 se observa la prueba ANOVA de las diferencias de medias para la puntuación $D$ de los y las estudiantes de distinta religiosidad de la muestra, que indica si existen diferencias entre las medias de la puntuación $D$ de los diferentes grupos.

\section{Tabla 5}

Prueba ANOVA de diferencias de medias para la variable de puntuación $D$

\begin{tabular}{cccccc}
\hline Casos & $\begin{array}{c}\text { Suma de } \\
\text { Cuadrados }\end{array}$ & df & $\begin{array}{c}\text { Media } \\
\text { Cuadrática }\end{array}$ & F & $\mathbf{p}$ \\
\hline Religiosidad & 0.718 & 3.000 & 0.239 & 1.453 & 0.235 \\
\hline Residual & 10.877 & 66.000 & 0.165 & & \\
\hline
\end{tabular}

Nota: la fuente es el Programa JASP — a partir de datos obtenidos de TAI aplicado a un grupo de estudiantes del INTEC en 2020-.

En la Tabla 5 se puede apreciar que el valor de $\mathrm{p}$ es igual a 0.235 . Como el valor de $\mathrm{p}$ es mayor a 0.05 , se concluye que no existen diferencias significativas entre la puntuación $D$ de estudiantes de distintos niveles de religiosidad. 
En la Tabla 6 se observan los descriptivos de la puntuación $D$-que indica preferencia racial automática- que presentan los y las estudiantes de distintas identidades políticas de la muestra, señalándose media, desviación estándar y tamaño de cada grupo.

Tabla 6

Descriptivos de puntuación D

\begin{tabular}{cccc}
\hline Identidad Política & Media & SD & N \\
\hline Moderadamente conservador & 0.373 & 0.322 & 4 \\
\hline Levemente conservador & 0.475 & 0.184 & 2 \\
Neutral & 0.287 & 0.416 & 41 \\
\hline Levemente liberal & 0.243 & 0.356 & 7 \\
\hline Moderadamente liberal & 0.362 & 0.544 & 12 \\
\hline Fuertemente liberal & 0.259 & 0.225 & 4 \\
\hline
\end{tabular}

Nota: la fuente es el Programa JASP — a partir de datos obtenidos de TAI aplicado a un grupo de estudiantes del INTEC en 2020-.

En la Tabla 6 se observa que la media de la puntuación $D$ en los y las participantes de identidad política levemente conservadora es de 0.475 ; en los de identidad política moderadamente conservadora es de 0.373; en aquellos/as de identidad política moderadamente liberal es de 0.362; en los de identidad política neutral es de 0.287; en los de identidad política fuertemente liberal es de 0.259 y en aquellos/as de identidad política levemente liberal la media es de 0.243.

En la Tabla 7 se observa la prueba ANOVA de las diferencias de medias para la variable puntuación $D$ de los y las estudiantes de distinta identidad política de la muestra, que señala si existen diferencias entre las medias de la puntuación $D$ de los diferentes grupos.

\section{Tabla 7}

Prueba ANOVA de diferencias de medias para la variable de puntuación $D$

\begin{tabular}{cccccc}
\hline Casos & $\begin{array}{c}\text { Suma de } \\
\text { Cuadrados }\end{array}$ & df & $\begin{array}{c}\text { Media } \\
\text { Cuadrática }\end{array}$ & F & $\mathbf{p}$ \\
\hline $\begin{array}{c}\text { Identidad } \\
\text { Política }\end{array}$ & 0.163 & 5.000 & 0.033 & 0.182 & 0.968 \\
Residual & 11.432 & 64.000 & 0.179 & & \\
\hline
\end{tabular}

Nota: la fuente es el Programa JASP — a partir de datos obtenidos de TAI aplicado a un grupo de estudiantes del INTEC en 2020-.

En la Tabla 7 se puede apreciar que el valor de $\mathrm{p}$ es igual a 0.968. Al ser el valor de p mayor a 0.05, se interpreta que no existen diferencias significativas entre la puntuación $D$ de estudiantes de distinta identidad política.

En la Tabla 8 se observan los descriptivos de la puntuación $D$ - que indica preferencia racial automática- que presentan los y las estudiantes de distintas clases sociales de la muestra, indicándose media, desviación estándar y tamaño de cada grupo.

\section{Tabla 8}

Descriptivos de puntuación D

\begin{tabular}{cccc}
\hline Clase Social & Media & SD & N \\
\hline Alta & 0.379 & 0.296 & 8 \\
\hline Baja & 0.340 & 0.562 & 9 \\
\hline Media & 0.287 & 0.401 & 53 \\
\hline
\end{tabular}

Nota: la fuente es el Programa JASP — a partir de datos obtenidos de TAI aplicado a un grupo de estudiantes del INTEC en 2020 -

En la Tabla 8 se puede observar que la media de la puntuación $D$ en estudiantes de clase alta es de 0.379 , en los de clase baja es de 0.340 y en aquellos/as de clase media es de 0.287. 
En la Tabla 9 se visualiza la prueba ANOVA de las diferencias de medias para la variable de puntuación $D$ de los y las estudiantes de distintas clases sociales de la muestra, que señala si existen diferencias entre las medias de la puntuación $D$ de los diferentes grupos.

\section{Tabla 9}

Prueba ANOVA de diferencias de medias para la variable de puntuación $D$

\begin{tabular}{cccccc}
\hline Casos & $\begin{array}{c}\text { Suma de } \\
\text { Cuadrados }\end{array}$ & df & $\begin{array}{c}\text { Media } \\
\text { Cuadrática }\end{array}$ & F & $\mathbf{p}$ \\
\hline $\begin{array}{c}\text { Clase } \\
\text { Social }\end{array}$ & 0.071 & 2.000 & 0.036 & 0.207 & 0.813 \\
\hline Residual & 11.524 & 67.000 & 0.172 & & \\
\hline
\end{tabular}

Nota: la fuente es el Programa JASP — a partir de datos obtenidos de TAI aplicado a un grupo de estudiantes del INTEC en 2020-.

En la Tabla 9 se observa que el valor de $\mathrm{p}$ es igual a 0.813 . Como el valor de $\mathrm{p}$ es mayor a 0.05 , se concluye que no existen diferencias significativas entre la puntuación $D$ de estudiantes de distintas clases sociales.

\section{Conclusiones y discusiones}

A continuación, se presenta la discusión de los resultados obtenidos en la presente investigación, cuyo objetivo general consiste en evaluar si existen manifestaciones de racismo implícito en un grupo de estudiantes del INTEC.

Esta investigación es de gran importancia, ya que estudia la nueva manera en que se manifiesta predominantemente el racismo en la actualidad en un contexto universitario; de manera que concientiza sobre este fenómeno que resulta más difícil de identificar.

En relación a si se presentan diferencias con relación al sexo en la existencia o no de racismo implícito en un grupo de estudiantes del INTEC, la investigación determinó que no existen diferencias significativas entre la puntuación $D$ de hombres y mujeres de la muestra utilizada, la cual determina la preferencia racial automática de estos y estas.
Wade (2013) señala que las inclusiones y exclusiones por razones de etnia se manifiestan también por medio de las relaciones de sexo/género. Quintero (2013) indica que en su estudio llevado a cabo con estudiantes universitarios de París y Bogotá se encontró una notoria mayor cantidad de mujeres que habían sido víctimas de racismo que de hombres, donde de las 775 situaciones relatadas, el $71 \%$ de estas fueron experimentadas por mujeres. Romero (2015) explica que durante los ańos de esclavitud la mujer afroamericana fue marginada tanto por blancos como por negros. Señala que los hombres blancos las discriminaban tanto por su grupo étnico como por su sexo y los hombres afroamericanos las excluían de la lucha por su libertad por ser considerada inferior.

A través de lo expresado por estos autores se intuiría que sí habrían diferencias significativas en la existencia o no de racismo implícito entre hombres y mujeres de la muestra. El hecho de que la historia explique que las mujeres eran discriminadas por hombres por razones de etnia y sexo deja la idea tanto de que una gran cantidad de mujeres han sufrido una doble discriminación, como de que muchos hombres discriminaron doblemente.

Sin embargo, se observa que los resultados de la investigación indican que en este grupo de estudiantes del INTEC no se observan diferencias significativas en la preferencia racial automática de ambos sexos. Esto no necesariamente indica que no las haya en el INTEC, ni mucho menos en República Dominicana, debido a que la muestra utilizada no es tan representativa; pero, sí describe e informa sobre cómo es la situación en un grupo de estudiantes de distintas edades, carreras, tonalidades de piel, ideas políticas, clases sociales, religiones y religiosidad. Este último factor es muy importante para tomar en cuenta cuando hablamos de racismo, debido a la relación que guardan entre sí.

Con relación a si se observan diferencias en la presencia o no de racismo implícito en un grupo de estudiantes del INTEC por su religiosidad, se encontró que no hay diferencias significativas entre la puntuación $D$ de estudiantes de distinta religio- 
sidad que componen la muestra, la cual determina su preferencia racial automática.

A pesar de estos hallazgos, Hall et al. (2010) señalan que usualmente quienes son religiosos o religiosas tienen mayor tendencia a tomar decisiones y actuar frente a otros $\mathrm{u}$ otras, dependiendo de si pertenecen o no a su grupo. Agregan que en cuanto una religión suela practicarse entre una determinada etnia, se va forjando la percepción de que personas de otros grupos étnicos no pertenecen a estos grupos religiosos.

Como vemos, estos autores sugieren que mientras mayor sea el nivel de religiosidad de una persona, posiblemente su nivel de racismo sea mayor. Ya sabiendo que los resultados indican que no hay diferencias significativas en la existencia o no de racismo implícito, según la religiosidad de este grupo de estudiantes, consideramos interesante destacar que la media de la puntuación $D$ de aquellas personas que se identifican como no religiosas indica que presentan una leve preferencia hacia personas blancas sobre negras, mientras la media del resto de los grupos — de un nivel de religiosidad mayor - indica una moderada preferencia hacia personas blancas sobre negras, a excepción de los fuertemente religiosos o religiosas, que sí tenían el mismo nivel de racismo de los no religiosos o religiosas, pero este grupo solo lo constituye un o una estudiante de la muestra - por lo que es el grupo de menor representatividad-y los demás grupos sí estaban compuestos por un número similar de estudiantes.

Otro factor que involucra ideales y creencias de las personas, que va relacionado al racismo, es la identidad política. Para esta investigación se utilizó una muestra de identidades políticas liberales, conservadoras y neutrales, ya que los ideales fundamentales que conllevan estas identidades se consideran poder estar relacionados con el racismo.

En cuanto a si se presentan diferencias en la existencia o no de racismo implícito en un grupo de estudiantes del INTEC según su identidad política, se encontró que no hay diferencias significativas entre la puntuación $D$ de los y las estudiantes de distintas identidades políticas de la muestra.
Garreta (2012) explica que el liberalismo político busca evitar los conflictos y tiene como objetivo que los ciudadanos y las ciudadanas se encuentren a favor de ideales lo más heterogéneos que sea posible con respecto a orientaciones morales, religiosas o filosóficas. A pesar de esto, Mazariegos (2006) indica que lo que propone el liberalismo se ve olvidado cuando se trata de afrontar decisiones sobre la ciudadanía de ciertas etnias, como ocurre con la indígena, donde se impone a personas indígenas el requisito de no ser ese ser humano inculto primitivo que imaginan los y las liberales.

Por otro lado, Rottenbacher et al. (2011) explican que la ideología política conservadora se opone a los cambios sociales y más bien prefiere ideas tradicionales y seguras para la organización de las instituciones y las personas, lo que generalmente viene con desigualdad. Además, manifiestan actitudes que demuestran preferencias por acciones jerárquicas que incluyen diferencias de poder entre grupos e individuos, agregando que suelen preferir la superioridad de alguien del propio grupo frente a alguien externo a este.

Según lo expresado por estos autores, se esperaría que la ideología política conservadora estuviese más relacionada con la discriminación racial que la ideología liberal; no obstante, a la vez, se afirma que en ambos casos se pudiesen encontrar actitudes racistas. En los resultados del presente estudio no se observan diferencias significativas en la existencia o no de racismo implícito según las identidades políticas de este grupo de estudiantes del INTEC. Empero, se considera importante señalar que la media de la puntuación $D$ de los y las estudiantes que se consideran conservadores indica mayor nivel de racismo implícito que la de aquellos y aquellas de identidad política liberal.

Otro punto por analizar que, aunque no constituye específicamente una manera de seguir doctrinas o ideologías, definitivamente involucra creencias y valores que lo llevan a constituir un factor íntimamente relacionado al racismo; se trata de la clase social. En torno a si hay diferencias en la existencia o no de racismo implícito en un grupo de estudiantes 
del INTEC, según sus clases sociales, se encontró que no existen diferencias significativas entre la puntuación $D$ de estudiantes de diferentes clases sociales de la muestra utilizada.

Cea (2009) indica que el racismo y la xenofobia, en muchos casos, reflejan un fuerte clasismo en las personas. Ipar \& Giller (2016) explican que durante los inicios del racismo moderno no se observaba un racismo exclusivo, que pretende erradicar poblaciones completas de una etnia, sino más bien un racismo inclusivo que viene con la creación de relaciones de poder y con la explotación de clase. Añaden que esto recae en un racismo de clase cuyo objetivo consiste en diferenciar a las poblaciones según los roles que se desempeñen en la actual división del trabajo.

Esto que expresan estos autores haría pensar que se observarían diferencias en la existencia o no de racismo implícito en las distintas clases sociales de la muestra; esto debido a que, si el racismo va muy relacionado a la clase social, principalmente este racismo más solapado, que se puede manifestar bastante en un racismo de clase, entonces posiblemente en las personas de distintas clases sociales — donde se podrían manifestar distintos tipos de actitudes clasistas - también se esperarían distintos niveles de racismo.

A pesar de esto, los resultados de esta investigación indican que no hay diferencias significativas en la existencia o no de racismo implícito entre los y las estudiantes de distintas clases sociales de la muestra utilizada; no obstante, es relevante resaltar que la media de la puntuación $D$ que indica la preferencia racial de los y las participantes, tanto pertenecientes a clase baja como a clase media, sugiere una leve preferencia automática hacia personas blancas sobre personas negras, mientras que la media de las puntuaciones de aquellos $y$ aquellas que pertenecen a una clase social alta indica una preferencia automática moderada hacia personas blancas sobre personas negras.

Por consiguiente, esta investigación sugiere que no se observan diferencias significativas ni en el sexo, ni en la religiosidad, ni en la identidad política, ni tampoco en la clase social en la existencia o no de racismo implícito en este grupo de estudiantes del INTEC. Sin embargo, sí se presentan manifestaciones de racismo implícito en este grupo de estudiantes del INTEC.

Un porcentaje muy bajo de la muestra seleccionada $-21.429 \%$ - denotó ligera o ninguna preferencia racial automática hacia personas blancas sobre personas negras. El resto - 78.572\%— sí presentó distintos niveles de racismo implícito hacia alguna etnia, siendo notoriamente mayor la preferencia racial automática hacia personas blancas sobre personas negras. Cabe destacar que el nivel de racismo implícito más predominante en este grupo de estudiantes es de preferencia automática moderada hacia personas blancas sobre negras.

Los cambios de normativas sociales en los últimos 50 años, que incluyen el rechazo y la recriminación de actitudes racistas, han venido con la aparición de modalidades más encubiertas y latentes de estos prejuicios ante las víctimas (Pascale, 2010). Espelt \& Javaloy (1997) expresan que las diferencias en la distribución del poder tanto político, como social y económico evidencian que existe un racismo que aunque puede ser menos intenso en la manera en que se manifiesta, no lo es en cuanto a consecuencias; estas incluyen discriminación en el trabajo, la vivienda, la escuela y la sociedad para las minorías étnicas.

En el nuevo racismo se desplaza el fenotipo hacia la cultura, donde se cambia el anhelo por alcanzar la pureza racial por el de conseguir una identidad cultural auténtica. Además, hay un desplazamiento de la desigualdad hacia la diferencia, donde el rechazo a inferiores se manifiesta con la evitación del otro o de la otra (Espelt \& Javaloy, 1997).

Para concluir, en esta investigación se pudo observar que sí hay presencia de racismo implícito en este grupo de estudiantes del INTEC. Una gran mayoría presentó una preferencia racial automática hacia algún grupo étnico, de manera muy mayoritaria en distintos niveles de preferencia hacia personas blancas frente a personas negras, siendo el nivel de preferencia automática moderada el más frecuente. Además, se observó que no hay diferencias en la existencia o no de racismo implícito según el sexo de este grupo de estudiantes; tampoco según su nivel de religiosidad, identidad 
política o clase social. No obstante, sí se observó existencia de racismo implícito en la media de la puntuación $D$ de cada uno de los grupos.

\section{Referencias}

Aguayo, H. \& Piña, J. (2015). Expresiones de racismo en una muestra de estudiantes universitarios. Sinéctica, revista electrónica de educación, (46), 1-21. https://sinectica.iteso.mx/index.php/ SINECTICA/article/view/600/629

Ayala, A. (2012). La utilización del Test de Asociación Implícita en los procesos electorales. Tribunal electoral del Poder Judicial de la Federación, 267-292. https://dialnet.unirioja.es/descarga/ articulo/4725555.pdf

Baronnet, B. \& Morales-González (2018). Racismo y currículum de educación indígena. Ra Ximhai, 14(2), 19-29. https://dx.doi.org/10.35197/rx. 14.02.2018.01.bb

Buraschi, D. \& Aguilar-Idáñez, M. (2017). Herramientas conceptuales para un antirracismo crítico-transformador. Tabula Rasa, (26), 171-191. https://doi.org/10.25058/20112742.193

Cea, M. (2009). La compleja detección del racismo y la xenofobia a través de encuesta: un paso adelante en su medición. REIS, (125), 13-45. http://www.reis.cis.es/REIS/PDF/REIS_ 125_011231144723167.pdf

Chirix, E. \& Sajbin, V. (2019). Estudio sobre racismo, discriminación y brechas de desigualdad en Guatemala. CEPAL. https://repositorio.cepal.org/ bitstream/handle/11362/44422/1/S1900068_ es.pdf

Cisneros, I. (2001). Intolerancia cultural: Racismo, Nacionalismo, Xenofobia. Perfiles Latinoamericanos, 10(18), 177-189. https://dialnet.unirioja. es/servlet/articulo?codigo $=2212253$

Comisión Económica para América Latina y el Caribe -CEPAL_- (2016). La matriz de la desigualdad social en América Latina. CEPAL, Naciones Unidas. https://www.cepal.org/sites/default/files/ events/files/matriz_de_la_desigualdad.pdf
Denis-Rosario, M. \& Paulino, E. (2016). Desmantelando la nación dominicana: confrontando desnacionalización, hilvanando la identidad nacional. Revista digital de historia y arqueología desde el caribe, (28), 1-6. http://dx.doi. org/10.14482/memor.28.8095

Dicló, L. (2019). La afrodescendencia en la sociedad dominicana: entre la blancofilia y la negrofobia [Tesis doctoral, Universidad Complutense de Madrid]. Repositorio E-Prints Complutense. https://eprints.ucm.es/50843/1/T40792.pdf

Espelt, E. \& Javaloy, F. (1997). El racismo moderno. Departamento de Psicología Social de la Universidad de Barcelona. https://www.ciudadredonda.org/admin/upload/IMG/pdf/El_ racismo_moderno.pdf

Garreta, M. (2012). Liberalismo político: justificación pública dentro y fuera de las fronteras de una democracia constitucional. Eidos, (17), 192-223. http://www.scielo.org.co/pdf/eidos/ n17/n17a08.pdf

Greenwald, A., McGhee, D., \& Schwartz, J. (1998). Measuring individual differences in implicit cognition: the Implicit Association Test. Journal of personality and social psychology, 74(6), 1464-1480. https://psycnet.apa.org/doi/ 10.1037/0022-3514.74.6.1464

Hall, D., Matz, D., \& Wood, W. (2010). ¿Why Don't We Practice What We Preach? A Meta-Analytic Review of Religious Racism. Personality and social Psychology Review, 14(1), 126-139. https:// doi.org/10.1177/1088868309352179

Hellebrandová, K. (2014). Escapando a los estereotipos sexuales racializados: el caso de las personas afrodescendientes de clase media en Bogotá. Revista de Estudios Sociales. (49), 87-100. http://dx.doi.org/10.7440/res49.2014.07

Hernández-Sampieri, R., Fernández, C. \& Batista, M. (2014). Metodología de la investigación (6ta ed.). McGraw-Hill. http://observatorio.epacartagena.gov.co/wp-content/uploads/2017/08/ metodologia-de-la-investigacion-sexta-edicion.compressed.pdf 
Ipar, E. \& Giller, D. (2016). ¿De qué racismo(s) somos contemporáneos en Argentina? La persistencia del racismo como desafío explicativo para la sociología. Methaodos.revista de ciencias sociales, 4(2), 258-273. https://dx.doi.org/10.17502/m. rcs.v4i 2.120

Johnson, M., Rowatt, W., \& LaBouff, J. (2010). Priming christian religious concepts increases racial prejudice. Social psychological and personality science, 1(2), 119-126. https://doi.org/10. 1177/1948550609357246

Marques-García, L. (2019). Desigualdades de género en República Dominicana 2018-2022. Banco Interamericano de Desarrollo _-BID_. https:// publications.iadb.org/publications/spanish/ document/Desigualdades_de_género_en_ República_Dominicana_2018_-_2020_es_ es.pdf

Mateos-Jiménez, A., Torres-Martínez, B. \& García-Fernández, B. (2018). Impacto de un relato en las percepciones de racismo y sexismo de escolares de Primaria. Ocnos: Revista de Estudios sobre lectura, 17(2), 55-66. https://doi.org/10.18239/ ocnos_2018.17.2.1570

Mato, D. (2017). Superar el racismo oculto e interculturizar las universidades. Experiencias, avances y desafíos. + E: Revista de Extensión Universitaria, 7(7), 188-203. https://doi.org/10.14409/extension.v0i7.7064

Mazariegos, J. (2006). El liberalismo-racismo y la occidentalización capitalista: los imaginarios sociales y el saber sobre lo indigena-campesino en Guatemala liberal (1893-1959). DEI, Departamento Ecuménico de Investigaciones. http://biblioteca.clacso.edu.ar/Costa_Rica/dei/20120712 012557/liberalismo.pdf

Montero J. (2019). Racialidad, racismo y emancipación de los afrodescendientes en el oriente cubano. Una mirada desde la región las tunas. Cuadernos intercambio sobre Centroamérica y el Caribe, 16(1), 1659-4940. https://doi. org/10.15517/c.a..v16i1.36549

Ortiz-Hernández, L., Compeán-Dardón, S.,Verde-Flota, E. \& Flores-Martínez, M. (2011).
Racismo y salud mental en estudiantes universitarios de la ciudad de México. Universidad Autónoma Metropolitana. https://www.scielosp.org/ article/spm/2011.v53n2/125-133/

París, M. (2002). Estudios sobre el racismo en América Latina. Politica y Cultura, (17), 289-310. https:// www.redalyc.org/pdf/267/26701714.pdf

Pascale, P. (2010). Nuevas formas de racismo: Estado de la cuestión en la psicología social del prejuicio. Ciencias Psicológicas, 4(1), 57-69. http//doi. org/10.22235/cp.v4i1.111

Pineda, E. (2018a). Los afrodescendientes ante el racismo latinoamericano: autorreconocimiento étnico y endorracismo. Revista da ABPN Associação Brasileira de Pesquisadores(as) Negros(as), 10(26), 10-26. https://www.aacademica.org/ estherpinedag/15.pdf

Pineda, E. (2018b). Las heridas del racismo: Efectos psicosociales de la discriminación racial en las personas afrodescendientes en América Latina. Iberoamérica Social, 54-72. https://www. academia.edu/38231719/Pineda-E._2018_._ Las_heridas_del_racismo_Efectos_psicosociales_de_la_discriminación_racial_en_las_ personas_afrodescendientes_en_América_ Latina

Quintero, O. (2013). Racismo y discriminación en la universidad: lecturas cruzadas de las sociedades francesa y colombiana a partir de la experiencia vivida por estudiantes negros en París y Bogotá [Tesis doctoral, Université Rennes 2]. https:// tel.archives-ouvertes.fr/tel-00807356/document

Quintero, O. (2014). El racismo cotidiano en la universidad colombiana desde la experiencia vivida por los estudiantes negros en Bogotá. Universitas humanistica, (77), 71-94. https:// doi.org/10.11144/Javeriana.UH77.rcuc

Restrepo, A. (2019). Racismo sistémico y antihaitianismo en la República Dominicana. La Factoría, la revista social, (61), 11-17. https://revistalafactoria.org/articulos/racismo-sistemico-antihaitianismo-republica-dominicana 
Romero, A. (2015). Raza y género: violencia en The Bluest Eye (1970) de Toni Morrison. Oceánide, (8), 1-13. https://dialnet.unirioja.es/servlet/ articulo? codigo $=6236595$

Rottenbacher, J., Espinosa, A. \& Magallanes, J. (2011). Analizando el Prejuicio: Bases ideológicas del Racismo, el Sexismo y la Homofobia en una muestra de habitantes de la ciudad de Lima-Perú. Psicología Política, 11(22), 225-246. https://dialnet.unirioja.es/descarga/articulo/3898832.pdf

Seda-Prado, J. (2003). La Cuestión Étnico-Racial en el Pensamiento de Carlos Esteban Deive. Revista Mexicana del Caribe, 8(15), 107-135. https:// www.redalyc.org/pdf/128/12881504.pdf

Soler, S. (2018). Racismo y educación. Educação em Revista, (34), 2-27. http://dx.doi.org/10. 1590/0102-4698192221

Tosi, J., Ledesma ,R., Poó, F., Montes, S. \& López, S. (2018). El Test de Asociaciones Implícitas (Implicit Association Test). Una Revisión Metodológica. Revista Iberoamericana de Diagnóstico y Evaluación- e Avaliação Psicológica. RIDEP, 1(46), 175-187. https://doi.org/10.21865/ RIDEP46.1.13
Velasco-Cruz, S. (2016). Racismo y educación en México. Revista Mexicana de Ciencias Políticas y Sociales, (226), 379-408. https://doi.org/10. 1016/S0185-1918(16)30015-0

Vinueza, J. (1999). ElRacismo en las Américasy el Caribe (1era ed.). Editorial Abya Yala. https://books. google.com.do/books?hl=es\&lr=\&id=YGtMpGYjb_MC\&oi=fnd \&pg=PA7\&dq=vinue$\mathrm{za}+$ racismo\&ots $=\mathrm{j} Y-\mathrm{J} 5 \mathrm{DCGhR} \& \operatorname{sig}=\mathrm{tM} 8 \mathrm{IPa}-$ cIttTOhH516EUcSz9ntxk\&redir_esc=y\#v=onepage $\& \mathrm{q}=$ vinueza $\% 20$ racismo $\& \mathrm{f}=$ false

Wade, P. (2013). Racismo, democracia racial, mestizaje y relaciones de sexo/género. Tabula Rasa, (18), 43-72. https://doi.org/10.25058/20112 742.138

Woldenberg, J. (1997). Racismo y Educación. Revista mexicana de ciencias politicas y sociales, 41(167), 153-160. http://dx.doi.org/10.22201/ fcpys.2448492xe.1997.167.49430 